\title{
El futuro de la filosofía de la mente
}

Ricardo Braun

El artículo presenta y discute los temas más relevantes de la filosofía de la mente que ocupan el interés de la filosofía contemporánea y que señalan el camino del futuro de la especialidad en los siguientes años. Adicionalmente, se trata de poner en relieve la función de la filosofia de la mente en la aclaración y avance del conocimiento de los fenómenos mentales. Se abordan los siguientes temas: la importancia de la filosofía en los estudios psicológicos; el dualismo en la actualidad y sus repercusiones en la concepción de lo mental desde una perspectiva fisicalista; el reduccionismo y eliminativismo contemporáneos; el individualismo y la constricción sobre la ciencia de la psicología; y, finalmente, la relación de la conciencia y el cerebro.

\section{Dualismo / Reduccionismo / Individualismo / Conciencia}

\section{The future of philosophy of mind}

In this essay the main topics in contemporary philosophy of mind are presented and discussed. They show the current mainstream in the philosophy of mind and the projection for the years to come. In addition, the function of philosophy of mind in the advancement in the understanding of mental phenomena is highlighted. The following topics are addressed: The importance of philosophy in psychology; contemporary dualism and its repercussions on the physicalist conception of the mental is addressed; reductionism and eliminativism are discussed; individualism and the constriction on the science of psychology; and lastly, the relationship between brain and consciousness

Dualism / Reduccionism / Individualism / Consciousness 
Existen acontecimientos que son fáciles de predecir, como la frecuencia de un péndulo simple dada su longitud y aceleración gravitatoria. Muchos sucesos estudiados en la física tienen un comportamiento esperable similar y por ello los físicos suelen ser envidiados en su capacidad predictiva de los fenómenos. En las disciplinas que estudian al ser humano nos encontramos con dificultades para hacer predicciones casi por definición, particularmente con las conductas complejas. La filosofía no es excepción. Cuando se estudia la historia de la filosofía nos encontramos con pensadores notables, cuya aparición no hubiera podido ser predicha sino solo explicada a partir del contexto en el que se situó. Por ello, no es posible hacer pronósticos sobre el tipo de pensador con el que nos encontraremos en el futuro ni con la dirección temática que abordará.

Sin embargo, una forma de visualizar el futuro filosófico, pero no garantizarlo, es estudiar el curso de las acciones presentes $-\mathrm{O}$ las más recientes- con el que podríamos hacer inferencias sobre un cierto patrón de conductas futuras. Esta metodología es consonante con la concepción wittgensteiniana acerca de nuestros pronósticos: "Cuando pensamos en el futuro del mundo queremos siempre decir el destino que llegará si sigue yendo en la dirección que vemos en la actualidad; no se nos ocurre pensar que ese trayecto no es recto sino curvo, cambiando constantemente de direcciones" (Wittgenstein, 1980: 31).

En este artículo quiero explorar algunos de los temas más trabajados en los últimos diez años en la filosofía de la mente para argumentar las razones por las cuales estos tópicos nos ocuparán el interés y esfuerzo en los siguientes años. Empezaré discutiendo brevemente los temas que están indirectamente relacionados con la filosofía de la mente, para luego abordar los siguientes: problema mente-cuerpo, el reduccionismo, el individualismo, la neurología; y, finalmente, la conciencia. La orientación del artículo estará restringida a la denominada filosofía analítica, que predominantemente caracteriza a la filosofía anglosajona, y en consecuencia a los autores que examino.

\section{EL FUTURO Y VALOR DE LA FILOSOFÍA EN EL ESTUDIO DE LA MENTE}

Tanto los filósofos como los no filósofos suelen preguntarse si el trabajo filosófico tiene realmente alguna significación en el progreso del conocimiento de las diversas disciplinas. Dos preguntas surgen, entonces: ¿cuál es la función que la filosofía desempeña en una disciplina?, y ¿cuál es el propósito que la filosofía tiene en el contexto de las investigaciones académicas de la disciplina?

Existe una actitud adversa a la filosofía que se traduce en considerarla como suntuaria, superflua o, peor, inú- 
til. La filósofa norteamericana Martha Nussbaum resume esta actitud así:

Se nos representa como flojos y oscuros: cuando no nos escapamos de tareas significativas, producimos trabajos que no interesan a nadie salvo a uno $\mathrm{u}$ otro, y en la mayoría de veces, ni siquiera a uno u otro. Las revistas académicas son vistas como depósitos sin valor de productos sin inspiración, fruto de la lucha por la permanencia en la carrera docente, la vida intelectual como una vida mecanizada... Se diría que sostener a tales oscuros personajes irresponsables es un desperdicio de los fondos públicos y privados (Nussbaum, 1995: 144).

Esta caricatura con que se presenta a la filosofía se debe, en parte, al desconocimiento de la conexión entre esta y las diferentes disciplinas en la resolución de problemas teóricos y la aparente indiferencia que suele tener la actividad filosófica con la vida práctica, al dedicarse a asuntos que no tienen impacto sobre los problemas de las ciencias. Por otro lado, hay un desconocimiento de los temas que preocupan hoy en día a la filosofía profesional.

Generalmente se recuerdan las clases de filosofía recibidas en pregrado, que suelen ser introductorias o abordan temas que fueron históricamente relevantes. Este recuerdo muchas veces no presenta la dinámica e importante actividad del pensamiento actual, que proporcionaría otra visión de la filosofía. El estudiante puede llevarse la impresión de que son temas llamativos quizás para las personas de la especialidad, pero que en su quehacer diario o en su quehacer profesional, tendrán poca o nula importancia. Por ejemplo, una clase introductoria puede presentar el tradicional problema del escepticismo en las respuestas de Descartes y Hume, representando antagonismos irreconciliables. El estudio de la duda universal e hiperbólica emprendida por Descartes parece un ejercicio propedéutico, con miras a analizar las fuentes justificatorias del conocimiento. Pero no parece psicológicamente necesario que una persona esté buscando realmente emprender una justificación tipo cartesiana en vista de la cantidad de conocimientos que tenemos acerca del mundo y de nosotros mismos. De ahí que se considere que la era de la epistemología escéptica (o su combate) estén superadas. Como dice Searle, en referencia a la duda de la posibilidad de justificación del conocimiento:

Hay algo absurdo en el pensador posmoderno cuando compra un boleto de avión por internet, sube al avión, trabaja con su computadora laptop en el curso del vuelo, baja del avión al llegar a su destino, toma un taxi que lo lleva a la sala donde ofrececerá una conferencia, en la que sostiene que, de una forma $u$ otra, no hay conocimiento verdadero, que la objetividad es cuestionada y que toda aspiración a la verdad y al conocimiento son en realidad formas encubiertas de aprovechamiento de poder (Searle, 1999: 2071).

El recuerdo que se tiene de la tarea filosófica como solución o reafirmación del escepticismo, entre otros 
temas, es más bien de orden histórico. La filosofía tiene asuntos pendientes que sí pueden concitar el interés de las disciplinas científicas. En particular, en la psicología, como veremos, la filosofía actual y la que probablemente siga en los siguientes años puede aportar en el esclarecimiento conceptual y en la normatividad de la actividad psicológica en aquellos dominios tanto tradicionales como más novedosos.

\section{EL DUALISMO}

Así como la era de la epistemología escéptica tuvo su tiempo, el dualismo de sustancias entre mente y cuerpo, o mente y cerebro tuvo el suyo, y no parece tener un lugar en la filosofía de la mente contemporánea y la futura. Sin embargo, otras formas de dualismo han aparecido en el siglo XX y continúan teniendo vigencia. Son formas de dualismo de atributos o también dualismos metodológicos (Margolis, 1984).

Hasta la década de 1960 reinaron en la filosofía de la mente formas de conductismo lógico (Carnap, 1986) o metodológico (Ryle, 2005) y teorías de la identidad mente-cerebro (Smart, 1991). Sin embargo, el advenimiento del modelo funcionalista computacional, que se originó con el desarrollo de la inteligencia artificial, marcó un claro distanciamiento con los dos modelos predecesores. En efecto, el funcionalismo computacional proponía un "dua- lismo de descripción" de los eventos mentales, manteniendo, en ese sentido, la autonomía de la psicología, su vocabulario y metodología. Es conocida la analogía de la mente como software y el cerebro como un hardware, entre otros posibles realizadores del programa. Básicamente, lo que se sostiene es que existe un isomorfismo funcional entre una persona que se encuentra en un estado mental $\mathrm{E}$ y una computadora digital en el mismo estado. Aun cuando tienen materiales físicos distintos $-\mathrm{y}$ por lo tanto con diferentes especificaciones físicas- tendrían la misma función, que es descrita en forma abstracta. El funcionalismo sigue teniendo vigencia, aunque notablemente modificado de la versión inicial sugerida por Putnam (1990).

Por ejemplo, nos encontramos con Dennett (1990), que sostiene que para explicar óptimamente la conducta humana debemos escoger entre tres posibles perspectivas o posturas: la postura física, la postura de diseño y la postura intencional. La primera asemeja la idea del hardware mencionado, la segunda tiene una connotación más bien teleológica: se analiza el comportamiento de acuerdo a cómo fue pensado que actuara, independientemente de su realización física. Sin embargo, para explicar la compleja conducta humana, debemos adoptar la postura intencional, en la que suponemos que los seres humanos se comportan siguiendo sus deseos y creencias, bajo una normativi- 
dad racional. Por ejemplo, cuando quiero explicar por qué fui a la refrigeradora, se recurriría a una historia de interacción causal entre mi deseo de tomar cerveza y mi creencia de que en la refrigeradora están las cervezas. En esta postura no se ha mencionado ni la configuración física ni el diseño del agente que está actuando.

Como se podrá reconocer, la postura de Dennett no desconoce la fisicalidad de los eventos mentales, pero atribuye un lenguaje y conexión causal típicos de explicaciones cartesianas. Por ello llama a nuestra actividad mental un tipo de "teatro cartesiano" (Dennett, 1990). La vena fisicalista de Dennett se entiende cuando sugiere que las descripciones que hacemos desde la postura intencional podrían ser eventualmente reducidas a descripciones de las otras posturas, pero que por razones metodológicas no es conveniente realizarlas. Precisamente, esta posibilidad de hacer una reducción a nivel ontológico, pero que por razones de conveniencia se prefiere mantenerla en la autonomía de la postura intencional, ha provocado que Dennett sea considerado un instrumentalista de la psicología popular.

Otro dualismo contemporáneo muy influyente es el de Davidson y su doctrina del monismo anómalo (Davidson, 1980). Como se advierte fácilmente, Davidson rechaza el dualismo substancial, pero argumenta que la intencionalidad de nuestras descripciones cotidia- nas y de la psicología científica no puede ser reducida a descripciones no intencionales. Nuestra interacción con otros seres humanos requiere que nos veamos como seres racionales que utilizan el aparato conceptual típico de la psicología popular, las creencias y los deseos y otras actitudes proposicionales. Para explicar la conducta humana -y algunas veces predecirla- atribuimos creencias y deseos a otras personas, utilizando el denominado "principio de caridad", que es una presuposición que los seres humanos son racionales, consistentes y coherentes con respecto a sus actitudes.

Como se dijo, Davidson es monista, de manera que ontológicamente los seres humanos, en principio, están hechos de lo mismo que toda la naturaleza física. Sin embargo, no debiéramos reducir el lenguaje de la psicología -mayormente intencional- a una descripción en un lenguaje físico, por ejemplo la descripción de los procesos cerebrales. La justificación para ello es que en el lenguaje de la física no hay lugar para los aspectos normativos de nuestras descripciones intencionales $\mathrm{y}$, además, la característica de lo mental es tal que evade cualquier representación vía leyes estrictas como encontraríamos en la física. De ahí la denominación de monismo anómalo. El problema, sin embargo, es que con esta postura Davidson condena a la psicología a una discontinuidad con las demás ciencias, por incapacidad para 
adecuarse al modelo de explicación científica basado en el uso de leyes de cobertura.

Searle ha propuesto también otra forma de dualismo metodológico, al que llama dualismo de propiedades físicas o dualismo de lo biológico (Searle, 1984). Sostiene que así como en el caso del agua, que tiene la propiedad del estado líquido que no encontramos en sus componentes, hidrógeno y oxígeno, en el caso de los eventos mentales, estos últimos surgen como una propiedad emergente a partir de los procesos cerebrales. Para Searle, la propiedad de lo mental surge solo en los seres biológicos, como los seres humanos y otros animales superiores y no, por ejemplo, a partir de máquinas, por más sofisticadas que sean. Este concepto de emergentismo biológico aparece como una crítica al funcionalismo computacional, al que Searle anteriormente había objetado con su conocido argumento del "cuarto chino", que intenta demostrar la diferencia entre una máquina sintáctica como las computadoras y una máquina semántica como las mentes humanas (Searle, 1984).

Las posiciones dualistas descritas son una muestra del debate contemporáneo en la filosofía de la mente, que explícitamente reconoce la incapacidad de las descripciones y explicaciones físicas de dar cuenta del fenómeno mental. Independientemente de la posibilidad de reducción que veremos a continuación, parece ser que o bien por razones instrumentales, o por razones esenciales, lo mental elude la factibilidad de ser descrito, explicado y predicho con las herramientas proporcionadas por el vocabulario y la metodología de las ciencias físicas. Si, como sostiene Searle, lo mental resulta ser un conjunto de propiedades emergentes, la tarea será explicar cómo pueden aparecer dichas propiedades a partir de propiedades físicas del supuesto sustrato físico.

Sin embargo, puede ser que si en los siguientes años, la filosofía analítica mantiene una postura monista, como la viene defendiendo por décadas, deberá producir una reconceptualización del concepto de lo físico, ya sea a través de un concepto de "modos fisicos" - como lo son la masa y la energía- o una forma de pluralismo ontológico de lo físico.

\section{REDUCCIONISMO Y ELIMINATIVISMO}

Siguiendo la metodología de la llamada "navaja de Ockam" (Entia non sunt multiplicanda praeter necessitatem, que quiere decir "Las entidades no deben ser multiplicadas más allá de lo necesario"), muchos filósofos abrazan el reduccionismo metodológico como receta para una economía ontológica y limpieza conceptual. El reduccionismo en lo mental se refiere a la necesidad de traducir, reemplazar o eliminar el lenguaje y metodología de la psicología 
popular hacia un lenguaje neurofisiológico, como un primer paso, para eventualmente reducirlo a un lenguaje de las ciencias físicas. En cierto sentido, el conductismo lógico -la traducción del lenguaje mentalista a lenguaje conductual- fue una estrategia reduccionista de principios del siglo $\mathrm{XX}$.

Pero la forma radical de reduccionismo es el eliminativismo, que aboga por la desaparición del lenguaje de la psicología popular, y también de la científica de nuestros días, por el lenguaje neurológico. Los eliminativistas más conocidos en la filosofía contemporánea son Paul y Patricia Churchland. Para los Churchland, la psicología popular ofrece un terriblemente inadecuado programa para la investigación, explicación y predicción del comportamiento humano y la vida mental. Por ello vislumbran una eventual eliminación, tanto del lenguaje como de la ontología acompañante de la psicología popular en vista del progreso de las neurociencias. Dice Paul Churchland:

La psicología popular no es parte de esta creciente síntesis. Sus categorías intencionales se erigen magníficamente solitarias, sin ninguna perspectiva de reducción en un corpus más extenso... La impotencia explicativa de la psicología popular y su largo estancamiento nos inspiran poca fe en que sus categorías puedan encontrarse claramente reflejadas en el marco conceptual de la neurociencia. Al contrario, se nos recuerda cómo la alquimia debe de haberse parecido, con el comienzo de la química elemental, a la cosmología aris- totélica cuando empezaba a articularse la mecánica clásica, o como la concepción vitalista de la vida, cuando tomaba cuerpo la química orgánica (1991: 604).

El pronóstico para la psicología tal como la conocemos es sombrío según Paul Churchland: "A la larga, en la ciencia, en los laboratorios, dejaremos de usar ese vocabulario de creencias y deseos. Podemos seguir usándolo en el mercado y en la mesa del comedor" (Churchland, 1995: 42).

Sin embargo, la oferta y pronóstico eliminativistas distan mucho de haberse hecho realidad. Y parte de la razón para que no se cumpla el pronóstico es que el lenguaje psicológico sigue siendo esencial en nuestras explicaciones en la psicología, tanto la cotidiana como la científica. Y no por razones de incapacidad de ver el mundo en forma material, o por impedimentos técnicos, sino más bien porque el lenguaje no reduccionista permite explicar mejor nuestra complicada vida mental, incluyendo nuestra racionalidad y nuestra conciencia. Querer reducir estas dos realidades a explicaciones neurológicas y de ahí a las físicas sería equivalente a pedir a un economista que explique el flujo de capitales o la inflación o nivel de empleo mediante una teoría basada en la física de partículas. Como dicen Wilson y Keil, haciendo eco de las diferencias en los objetivos y profundidad de una explicación: "Tu explicación de por qué llegaste tarde a la casa para comer y la prueba matemá- 
tica de un teorema tienen poco en común" (1999: 140).

Los siguientes años nos dejarán ver cuál será el estatus que tendrá la llamada psicología popular frente a la psicología neurológica. Pero todo hace pensar que las posiciones reduccionistas y eliminativistas continuarán dentro de la filosofía de la mente futura, pero tendrán que elaborar nuevas propuestas porque las actuales no parecen superar el escollo tal como lo presenta Fodor:

\begin{abstract}
Aun si pudiéramos prescindir de ella [la psicología popular] en principio, esto no sería ningún argumento para prescindir de ella... Lo relevante es si vale la pena defender su eliminación de hecho. Y aquí la situación es absolutamente clara. No tenemos ninguna idea de cómo explicarnos nosotros a nosotros mismos, a excepción de un vocabulario que está saturado de psicología de la creencia y deseo. Uno está tentado de usar un argumento trascendental: lo que Kant dijo a Hume acerca de los objetos fisicos funciona, mutatis mutandis, para las actitudes proposicionales; no las podemos dejar ir porque no sabemos cómo (1985: 9-10).
\end{abstract}

\section{EL INDIVIDUALISMO Y LA PSICOLOGÍA}

El individualismo en la filosofía de la mente es una visión que ocupa un espacio importante en la discusión filosófica contemporánea acerca de la psicología, porque tiene diversas implicancias en la naturalización de la intencionalidad, la autonomía de la psicología, la superveniencia de lo mental sobre lo físico, la naturaleza de la causación mental, la viabilidad de la psicología popular y la forma como se conciben las ciencias cognitivas.

El concepto de individualismo aplicado a la psicología se refiere a la manera como los estados mentales deben ser individuados, clasificados o taxonomizados. Según Burge, el individualismo sostiene que los estados mentales son "tales que no hay una relación individuativa fundamental entre el que un individuo se encuentre en estos estados y la naturaleza del entorno físico o social del individuo" (1986: 3). De acuerdo con esta definición, la forma en que los estados mentales son taxonomizados no están afectados por los factores externos al individuo. Esta tesis también es llamada solipsismo metodológico (Fodor, 1980). Por lo tanto, dos sujetos que comparten todas las propiedades físicas intrínsecas deben ser taxonomizados bajo las mismas clases naturales. Para que los estados mentales sean de diferente clase, deben supervenir sobre diferentes propiedades físicas intrínsecas. O sea, la identidad física intrínseca supone identidad psicológica.

Esto significa que para propósitos de la explicación psicológica, la individuación de los estados mentales no presupone nada en particular acerca del mundo externo del individuo que tiene esos estados. Para el individuo que puede ser engañado constantemente por el genio maligno cartesiano, en el fondo, no habría diferencia si el mundo externo 
no existiera porque siempre tendría estados mentales causalmente eficaces, puesto que lo que explica el comportamiento de una persona no es cómo es el mundo externo realmente, sino cómo lo concibe la persona. Esta forma de concebir la clasificación de los estados mentales tiene mucha repercusión en el desarrollo de las ciencias cognitivas. El individualismo, como vemos, proporciona pautas para la investigación psicológica teórica. Como dice Stich: "Cualquier diferencia entre los organismos que no se manifieste como diferencia en los estados físicos internos, debiera ser ignorada por una teoría psicológica... Los acontecimientos históri$\cos$ y ambientales serán psicológicamente relevantes en tanto influyan en el estado físico interno actual del organismo" (1983: 164-165).

Una forma de entender el problema del individualismo es apelar a la clasificación de internalismo/externalismo. Los individualistas serían internalistas con respecto a los contenidos mentales, considerando que los significados se encuentran dentro de la cabeza. Los no individualistas, también llamados externalistas, afirman que los significados se encuentran en el exterior y no "dentro de la cabeza". El famoso ejemplo de Putnam (1990) de los gemelos o Doppelgängers nos invita a cuestionar el internalismo. Supongamos que en una Tierra gemela ( $\mathrm{Tg}$ ) existe un sujeto $\left(\mathrm{D}_{2}\right)$ que comparte todas las propiedades físicas intrínsecas con otro sujeto
$\left(D_{1}\right)$ en nuestra Tierra (T). Como son tierras gemelas, las dos tienen las mismas características fenoménicas, es decir, cuando $\mathrm{D}_{1}$ y $\mathrm{D}_{2}$ perciben su entorno, las propiedades fenoménicas son las mismas, en consecuencia, pueden tener las mismas representaciones de lo externo. Sin embargo, hay un detalle: como sabemos, el agua en $\mathrm{T}$ es $\mathrm{H}_{2} \mathrm{O}$, pero en $\mathrm{Tg}$ el agua es más bien XYZ. Como se dijo, las propiedades fenoménicas son idénticas, las dos aguas comparten sus propiedades perceptibles, pero su fórmula química es distinta. Según Putnam, y en contra del individualismo, por más que $\mathrm{D}_{1} \mathrm{y}_{2}$ tengan las mismas propiedades físicas intrínsecas, el contenido (significado semántico) y la referencia de las palabras y proposiciones no dependen de lo que está "dentro de la cabeza" sino más bien "fuera de ella". Cuando $\mathrm{D}_{1}$ usa la palabra "agua" se estará refiriendo a $\mathrm{H}_{2} \mathrm{O}$ y no XYZ. $\mathrm{D}_{1}$ puede desconocer el contenido químico del agua, pero, de acuerdo con el externalismo, eso no le previene que cuando usa el término "agua" se está refiriendo a $\mathrm{H}_{2} \mathrm{O}$. $\mathrm{Si}$ hubiera indicado al XYZ y dicho "Eso es agua" hubiera dicho algo falso con relación al contenido referido. De forma similar, si $\mathrm{D}_{2}$ usa el término "agua" se estaría refiriendo correctamente a XYZ y no a $\mathrm{H}_{2} \mathrm{O}$.

Las ciencias cognitivas trabajan bajo el paradigma informativo de la psicología y conciben por lo tanto que toda información es representable $y$, en 
consecuencia, manipulable en el nivel cerebral. Una posición como el individualismo establece restricciones taxonómicas. Entonces, en la taxonomía utilizada en las ciencias cognitivas, los individualistas considerarían que es innecesario tomar en cuenta el entorno al momento de clasificar las clases naturales psicológicas. Los estados mentales, como dicen algunos, están "en la cabeza" y no "fuera de ella". En consecuencia, los valores de verdad o falsedad en el significado de las cosas no requieren ser independientes de lo que está "fuera o dentro de la cabeza", como, por contraste, sostienen los adversarios.

Las restricciones de las que estamos hablando son referidas a la categorización de los estados y procesos mentales en la explicación psicológica. El individualismo especifica un conjunto de condiciones necesarias mínimas para las explicaciones adecuadas. Por ejemplo, con respecto a los contenidos, los individualistas, como hemos visto, consideran que lo que está fuera de la mente de la persona es irrelevante para la psicología. Independientemente de cómo es el mundo en comparación con la forma como es representado por el individuo, la psicología debe categorizar los contenidos tal como los representa el individuo y no por las relaciones del sujeto con el mundo externo. Lo que importa es cómo el sujeto representa el mundo, no cómo el mundo es en realidad. Como respuesta al experimento mental de los Doppelgängers, los individualistas sostendrán que lo que importa es cómo los Doppelgängers hayan representado del agua, que es líquida, que es medio azul, que hace lagos y ríos, etcétera. El saber si es $\mathrm{H}_{2} \mathrm{O}$ o XYZ no influye en su psicología particular. La psicología del individualista se centra y culmina en lo interno.

Puesto que para la explicación científica los roles causales son imprescindibles, el individualista sostiene que solo los estados internos físicos - estados cerebrales presumiblementepodrían tener un rol explicativo incluso entre los inputs y outputs periféricos (Devitt, 1990: 377). Los externalistas, por su lado, sostienen que para la ciencia cognitiva, los aspectos externos son decisivos en la elaboración de las representaciones internas, particularmente las representaciones que involucran la sensación y la percepción, puesto que tienen un rol imprescindible en la información sobre el entorno: tendría que haber un isomorfismo funcional entre un aspecto del ambiente y el proceso que el cerebro elabora de este, de lo contrario no nos explicaríamos las conductas adaptativas a entornos desconocidos. Por otro lado, es parte de la ciencia cognitiva el estudio de la competencia lingüística semántica. Si el externalismo es correcto, entonces los significados de las pala- 
bras usadas por un hablante dependerán, al menos parcialmente, de sus relaciones con el ambiente físico y social. En ese sentido, la tesis de Putnam sería reivindicada y el conocimiento semántico del hablante rebasaría el estudio de los estados físicos intrínsecos.

Lo interesante que resulta la discusión internalismo/externalismo en nuestros días no se restringe al campo de lo mental, y esto es relevante, porque demuestra la importancia que algunos temas filosóficos pueden tener en la investigación científica de diferentes disciplinas, y que cuestionaría la suposición de que la filosofía es una tarea aislada y solipsista. En las ciencias sociales se ha utilizado tradicionalmente el nombre de "individualismo metodológico" para referirse a metodologías como la teoría de la elección racional, que es típicamente individualista; es decir, se privilegia el rol de los individuos y lo que les es intrínseco para las explicaciones de fenómenos sociales. En la biología vemos cómo el individualismo es un tema que aparece en el debate sobre los niveles o unidades de la selección natural. El individualismo considera que el organismo es la unidad más grande en la que opera la selección natural. Como vimos anteriormente, las posiciones individualistas normarán las restricciones en la tarea explicativa y predictiva de sus respectivas disciplinas (Wilson, 2004).

\section{MENTE, CONCIENCIA Y CEREBRO}

Un último tópico que quiero resaltar es la investigación filosófica de la mente contemporánea con respecto al cerebro. El interés de los filósofos por el cerebro no es nuevo. Podemos recordar los esquemas del cerebro hechos por Descartes para defender su dualismo sustancial. Pero con los avances impresionantes de las neurociencias, los filósofos siguen encontrando cuestiones que requieren una aclaración conceptual, actividad típica de la filosofía.

Resolver preguntas empíricas acerca del cerebro corresponde a la neurociencia. Es tarea de la neurociencia, por ejemplo, explicar las condiciones neurales que permiten la función perceptiva, cognitiva y volitiva. Se confirmarán las teorías explicativas de esas funciones por medio de investigaciones experimentales. Pero existen preguntas conceptuales, por ejemplo el concepto de lo mental, de la memoria o del pensamiento y su relación con otros conceptos, como la relación entre lo psicológico y lo neural, entre lo mental y lo conductual que podrían ser mejor abordadas por la filosofía.

El cerebro se nos presenta como una de las fronteras más difíciles de conceptualizar con relación a lo mental, puesto que, como tradicionalmente se ha concebido, los términos y conceptos utilizados -tanto para el cerebro como para lo mental- aparecen como irreconciliables. Felizmente, con el fin 
del dualismo, hemos podido aceptar que los fenómenos mentales son fenómenos naturales más emparentados con lo biológico y no una esfera metafísicamente distinta que hace inaccesible cualquier tipo de comprensión cerebral de los acontecimientos mentales, en especial el de la conciencia. Es decir, ¿cómo llegamos a tener conciencia del complejo neuronal?

Uno de los primeros problemas por resolver, y que nos servirá como ilustración de esta sección, es el de la tradicional dicotomía entre lo "objetivo" y lo "subjetivo" que subyace a la relación cerebro y conciencia. Generalmente, cuando hablamos de "objetividad" nos referimos al conocimiento en tercera persona, típico de las ciencias físicas y que permite hacer observaciones empíricas, públicas y replicables. Pero la conciencia se nos aparece como fenoménica, en el sentido de que tenemos acceso privilegiado a los contenidos, experimentamos en primera persona nuestros actos mentales y tenemos una suerte de incorregibilidad acerca de nuestra experiencia consciente. Este problema lo resume Nagel en su conocido y sugerente título "¿Qué se siente ser un murciélago?" (1974). Según Nagel, para sentir como murciélago hay que ser murciélagos y tener la experiencia fenoménica que solo un murciélago podría tener. Análogamente, solo puedo tener la experiencia de sentir como yo, siendo yo, entonces no es posible identificar los hechos cerebrales con los hechos mentales, porque uno es objetivo y el otro es subjetivo.

Para Searle (1999), el problema de la dicotomía radica en que las categorías que se usan son obsoletas. Hablar de objetividad y subjetividad como categorías excluyentes cierra a priori la posibilidad de estudiar la conciencia desde el cerebro. Para Searle, la conciencia es, por definición, subjetiva, en el sentido de que para que exista un estado de conciencia tiene que ser experimentado por algún sujeto consciente. Concibe la conciencia como una ontología de primera persona que existe solo para el punto de vista humano, un "yo" que puede tener esa experiencia. Sin embargo, esta distinción ha alimentado la convicción de que las ciencias solo deben estudiar lo objetivo y que la conciencia, por ser subjetiva, no tiene lugar en el conjunto de conocimientos científicos.

Sin embargo, la subjetividad es un modo de existencia, que si bien es cierto que puede ser distinguible de otros modos de existencia, no elude su estudio científico. La denominada objetividad epistémica, valorada tradicionalmente, significa un esfuerzo por conocer las cosas, estableciendo proposiciones que sean potencialmente verdaderas. Pero esta objetividad epistémica no negaría la posibilidad de estudio de la subjetividad ontológica de la subjetividad. Eso supondría que la "privacidad" de la conciencia no hace 
imposible una ciencia de la conciencia. Precisamente esa parece ser la tarea en los siguientes años en el campo de la conciencia. $\mathrm{O}$ al menos eso espera Searle.

Finalmente, en su libro Philosophical Foundations of Neuroscience (2003), el neurocientífico M. Bennett y el filósofo Peter Hacker han cuestionado severamente algunas de las presuposiciones de las neurociencias con respecto a lo mental. El argumento central es una crítica a la concepción del cerebro como responsable de los atributos psicológicos, a lo que llaman una "falacia mereológica". La mereología es el estudio lógico del todo y sus partes. Se comete esta falacia cuando se cree que el cerebro, parte del cuerpo humano, es responsable de la actividad mental: los predicados psicológicos, que solo pueden ser aplicables a los seres humanos (u otros animales) como un todo, no pueden ser aplicados inteligiblemente a sus partes, como el cerebro.

Bennett y Hacker sostienen que la atribución de predicados psicológicos al cerebro es una cuestión, en primer lugar, filosófica y no tanto neurológica, puesto que es una pregunta conceptual. La convicción que comparten es que el cerebro, como tal, no es el sujeto apropiado para los predicados psicológicos. Esta posición contradice frontalmente la posición de autores como Damasio, quien sostiene que el cerebro puede "decidir" en segundos; Marr, que sos- tiene que nuestros cerebros "son capaces de representar información", o Crack, cuando afirma que el "cerebro cree y hace la mejor interpretación de acuerdo con su experiencia previa (Bennett y Hacker, 2003, cap. 3). En cambio, Bennett y Hacker afirman que adscribir predicados psicológicos al cerebro conduce a acrecentar la confusión. Y si lo hacen los neurólogos y científicos es por una incorrecta extrapolación de sus métodos y vocabulario. Lo que los neurólogos pueden hacer es descubrir las condiciones neurales, y la posibilidad del ejercicio de los poderes distinguiblemente humanos del pensamiento y el razonamiento. Esto 1o hacen por correlación entre los fenómenos neurales y las capacidades psicológicas. Pero, advierten, lo que no se puede hacer es volver a emplear nuestras explicaciones psicológicas en términos de razones, intenciones, propósitos, valores, reglas y convenciones por explicaciones neurológicas. Por ejemplo, no se puede pretender explicar cómo una persona piensa por referencia única al cerebro o a alguna parte del cerebro. En suma, no se pueden adscribir atributos psicológicos a nada menos, que a una persona como un todo y no sus partes. Es una persona la que percibe, y no parte de su cerebro: es una persona la que piensa y razona, no su cerebro.

Como se advertirá, esta última publicación está en espera de las reacciones de los implicados, particular- 
mente los neurocientíficos aludidos expresamente en la obra de Bennett y Hacker. Lo que nos demuestra, una vez más, la intensa actividad que nos tendrá ocupados en los siguientes años con respecto a la aclaración conceptual en el campo de las neurociencias.

\section{CONCLUSIONES}

Se puede ver que la filosofía está muy activa en el tratamiento del problema de lo mental y la tendencia a continuar de esa forma se evidencia con las investigaciones actuales. El fin del dualismo de sustancia no ha significado el fin del dualismo como un todo. Nuevas formas de dualismo han cobrado importancia como resultado de la incapacidad de los modelos monistas de dar cuenta de la complejidad de la actividad mental humana, siendo las más representativas el emergentismo de Searle, el monismo anómalo de Davidson y la perspectiva intencional de Dennett. El reduccionismo y eliminativismo, aun cuando tienen defensores, todavía no nos dan una historia más o menos creíble para pensar en la posibilidad de dejar nuestras categorías psicológicas por las categorías neurológicas o físicas. El individualismo, que es compartido con otras disciplinas científicas, en particular la biología, determinará en los siguientes años los límites de las formas de explicación en la psicología y en la ciencia cognitiva. Finalmente, aunque siempre se elude el tema de la conciencia humana, una tarea pendiente, que ya está siendo pensada, es la disolución de la dicotomía objetivo-subjetivo, que se pensó que era válida para relacionarla con el cerebro y la conciencia. Nuevas investigaciones en filosofía y en neurología nos permitirán resolver parte de ese tema, como el de la aplicación de los conceptos mentales o psicológicos en la neurociencia.

\section{REFERENCIAS}

Bennett, M. R. \& Hacker, P. M. S. (2003). Philosophical Foundations of Neuroscience. Malden, MA.: Blackwell.

Burge, T. (1986). Individualism and psychology. Philosophical Review, 95, 3-45.

Carnap, R. (1986). Psicología en lenguaje fisicalista. En: A. J. Ayer (Ed.). El positivismo lógico. México: Fondo de Cultura Económica.

Churchland, P. (1991). Eliminative materialism and the propositional attitudes. En: Rosental, D. (Ed.). The nature of mind. Nueva York: Oxford University Press.

Churchland, (1995). Neural Networks and Commonsense. En: Baumgartner, P. \& Payr, S. (Eds.). Speaking Minds: Interviews with Twenty Eminent Cognitive Scientists. Princeton: Princeton University Press. 
Davidson, D. (1980). Essays on Actions and Events. Oxford: Oxford University Press.

Dennett, D. (1990). The Intentional Stance. Cambridge, MA.: MIT Press.

Devitt, M.(1990). A Narrow Representational Theory of the Mind. En: Lycan, W. (Ed.). Mind and Cognition. Cambridge, MA.: Basil Blackwell.

Fodor, J. (1980). Representations. Cambridge, MA.: MIT Press.

Fodor, J. (1985). Fodor's guide to mental representations. Mind, 94, 77110.

Margolis, J. (1984). Philosophy of Psychology. Nueva Jersey: Prentice-Hall.

Nagel, T. (1974). What Is it Like to Be a Bat? Philosophical Review, 4, 435-50.

Nussbaum, M. (1995). Proceedings and Addresses of the American Philosophical Association, 69(2).

Place, U.T. (1956). Is Consciousness a brain process? British Journal of Psychology, 47: 44-50.

Putnam, H. (1990). The nature of mental states. En: Lycan, W. (Ed.), Mind and cognition. Oxford: Blackwell.

Ryle, G. (2005). El concepto de lo mental. Barcelona: Paidós.
Searle, J. (1984). Minds, brains and science. Cambridge, MA.: Harvard University Press.

Searle, J. (1992). The rediscovery of the mind. Cambridge, MA.: MIT Press.

Searle, J. (1999). The future of philosophy. Philosophical Transactions of the Royal.Society B: Biological Sciences, 29, 2069-2080.

Smart, J.J.C. (1963/1991). Sensations and brain processes. En R. Rosenthal (Ed.). The nature of mind. Nueva York: Oxford University Press.

Stich, S. (1983). From folk psychology to cognitive science. Cambridge, MA.: MIT Press.

Wilson, R. (1995). Cartesian Psychology and Physical Minds: Individualism and the Sciences of the Mind. Cambridge: Cambridge University Press.

Wilson, R. (2004). Recent Work in Individualism in the Social, Behavioral and Biological Sciences. Biology and Philosophy, 19, 397-423.

Wilson, R. \& Keil, F. (1999). The MIT Encyclopedia of the Cognitive Sciences. Cambridge, MA.: MIT Press.

Wittgenstein, L. (1980). Culture and value. Oxford: Basil Blackwell. 